\title{
OBSERVACIONES SOBRE UN EMPERADOR CRISTIANO: FI. Jul. CONSTANTE
}

\author{
ELISA GARRIDO GONZALEZ \\ Universidad Autónoma de Madrid
}

\begin{abstract}
La vida del hijo menor de Constantino el Grande, Fl. Jul. Constante, tiene planteada una serie de cuestiones aún sin resolver; por tal razón, este trabajo constituye un estudio sobre toda la etapa anterior a su toma del poder en todo el territorio occidental del Imperio, con la pretensión de ofrecer una explicación verosímil de un conjunto de hechos controvertidos, a la luz de la documentación disponible. En definitiva, se analizan temas como la instrucción recibida por Constante, su nombramiento como césar; igualmente se estudia de qué manera le afectó la distribución territorial del año 335 realizada por su padre y el posterior reparto acordado con sus hermanos en el 337; cómo se desarrolló la masacre de la mayoría de los miembros de la familia constantiniana, tras la muerte de Constantino y cómo surgió el conflicto con su hermano mayor Constantino II, cuyo resultado fue la implantación de su autoridad en toda la pars occidentis durante una década.
\end{abstract}

There are some unresolved problems concerning the life of the youngest son of Constantine the Great, Fl. Jul. Constante. This paper focuses on the period before his leading the occidental part of the Empire. We intend to offer a sound explanation of the whole range of relevant facts in the light of the available documentation. We analize subjects such as the intruction received by Constantine, his appointment as Caesar, the way in which the territorial distribution carried out by his father in 335 affected him and the later distribution arranged between his brothers in 337 . We also investigate how the massacre of the majority of the constantinian family developed itself, after Constantine death. Finally, we study how originated the conflict with his elder brother Constantine the 2 nd, that gave as a result the consolidation of his authority over the whole pars occidentis during a decade.

Constantino el Grande murió el 22 de mayo del año 337; a los tres meses, el 9 de septiembre, sus tres hijos: Constantino, Constancio y Constante, se proclamaron Augusti (Anon. Val., 35; AUR. VICT., Epit., 41, 20; EUS. Vita Const., 4, 51; ZOS. 2, 40-1; EUTR., Brev., 10, 9). Aquí me interesa estudiar de cerca al menor de ellos, Flavio Julio Constante, ya que este emperador parece haber sufrido en la historiografia moderna una damnatio memoriae, debido a que su vida, obra política y período de gobierno prácticamente no han despertado ningún interés entre los historiadores (1). Este hecho

(1) Hay estudios en los que, de alguna manera, Fl. Jul. Constante está incluido, cfr. BARNES, 1975, 325-333; BARNES, 1978, 53-75; BARNES, 1980, 160-166; BURCKHARDT, 1982, reimp.; CHASTAGNOL, 1982; DEMOUGEOT, 1979, 229-253; FORTINA, 1955; GIGLI, 1959; HAUSCHILD, 1966, 8692; KRAFT, 1958; MAURICE, 1911; MOREAU, 1959, 179-184; PALANQUE, 1938, 241-250; PALANQUE, 1944, 47-64; PALANQUE, 1955, 257-263; PIGANIOL, 1972 2; SEECK, 1898, 17-65; SEECK, 1919; SEECK, 1901, 948-952; THIRION, 1965, 5-21; TOYNBEE, 1944; VOGLER, 1979. 
causa, cuando menos, extrañeza ya que Constante durante una década completa ejerció la autoridad suprema en todos los territorios que comprendían el occidente imperial; ello significa que durante un período de tiempo considerable tuvo bajo su poder la mitad del Imperio; a pesar de lo cual, ha merecido una atención menor que otros emperadores bajoimperial. El evidente desinterés en la investigación actual manifestado para con este reinado es, en realidad, una consecuencia clara de la misma actitud sostenida desde la Antigüedad, pues no contamos por ejemplo con ninguna obra biográfica sobre Constante, o con algún o algunos documentos en que se nos proporcione un informe detallado respecto de dicho reinado. Así pues, contamos únicamente con noticias aisladas y dispersas en las obras de los autores de la época o posteriores como Zósimo, Sócrates, Atanasio, Ammiano, etc.; asimismo nos han llegado pocas inscripciones y monedas y conocemos algunas leyes suyas en el CTh, quizá la documentación más interesante (2).

El motivo de este silencio documental resulta ciertamente difícil de explicar, aunque ésta sea una circunstancia que se da y encontramos con bastante frecuencia en el mundo antiguo. No obstante, parece extraño que en un período de gobierno tan extenso no hubiera surgido algún biógrafo, panegirista o, en fin, cualquier otro tipo de cronista; desgraciadamente no fue así, por lo cual, en estos momentos y en el estado actual de la investigación ésta es la realidad de la que debemos partir (3).

A pesar de todo ello, está claro que se han podido esbozar las líneas generales de la vida de este emperador, así como de su actividad política. Ahora bien, igualmente es cierto que persisten aún muchas incógnitas y hechos controvertidos objeto de polémica para la investigación moderna; por lo cual, se hace necesaria, en la actualidad, una exposición de conjunto de toda esta problemática.

En primer lugar, se ha manifestado ya una inseguridad con respecto de la fecha de nacimiento de nuestro emperador, ya que se le puede asignar bien el año 320 o el año 323. Esta disparidad cronológica tiene su origen en las fuentes que indican, en realidad, la edad que tenía en el año de su muerte, el cual está bien establecido en el 350 (AUR. VICT., Epit., 41, 23; EUTR. Brev., 10, 9, 4; HIERON. Chron., 237 C; JUAN CRISOSTOMO, in epist. ad Philipp., 4, 5; JUL. Or., 2, 26 C; 2, 5 D; MALALAS, 325 Bonn; Chron. Min. 1, 237; ZON. 13, 6; ZOS. 2, 42; SOCR. HE 2, 25, 7; SOZ. HE 4, 1).

(2) La dispersión de las fuentes obliga a una precisa recopilación: AMM. 15, 5, 16;16,7,5;20,1,1; $20,11,3 ; 21,6,2 ; 27,8,4 ; 28,3,8 ; 30,7,5 ;$ Anon. Val. 35; ATHAN. Apol. c. Arian. 51-57; ATHAN. Apol. c. Const. 2-15; ATHAN. Hist. Ar. 69; ATHAN. Apol. Sec. 87, 4; ATHAN. Synod. 25; ATHAN. Festal Index 17; AUG. c. Cresc. 3, 49; AUR. VICT. Epit. 41, 19-24. AUR. VICT. Caes. 41, 13-24; AUS. Commen. Prof. Burdig 16, 15; CJ 1, 3, 1; 3, 26, 6; 10, 48, 7; Chron. Min. 1, 232-238; CTh 2, 6, 5; 2, 7, 3; 3, 5,$7 ; 7,1,2 ; 7,9,1 ; 8,2,1 ; 8,7,3 ; 9,7,3 ; 9,17,1 ; 9,21,6 ; 9,23,1 ; 9,24,2 ; 10,10,4 ; 10,10,5 ; 10,10,6$; $10,10,8 ; 10,10,7 ; 10,14,2 ; 10,14,3 ; 10,15,3 ; 11,12,1 ; 11,1,4 ; 11,7,6 ; 11,16,5 ; 11,30,23 ; 11,36,4$; $12,1,3 ; 12,1,24 ; 12,1,25 ; 12,1,26 ; 12,1,27 ; 12,1,29 ; 12,1,31 ; 12,1,36 ; 12,1,38 ; 12,6,5 ; 12,9,29$; $16,2,8 ; 16,8,1 ; 16,8,6 ; 16,9,2 ; 16,10,2 ; 16,10,3 ; 16,10,4 ; 15,1,5 ;$ EUN. V. Soph . 6, 3, 8; 90; EUS. Vita Const. 4, 40-68; EUS. Laud. 3; EUTR. Brev. 10, 6; 10, 9-2-4; FIRM. MAT. de err. prof. rel. 28, 6; Frag. Vat. 35; HIER. Chron. 2356, 2358, 2866; HILAR. Frg. Hist. 2, 21; CIL 3, $12330=$ ILS 8944; CIL 2, $6029=$ ILS 725; CIL 3, 4180=ILS 727; CIL 3, 2829; ILS 726; ILS 724; CIL 11, $5265=$ ILS 705; CIL 3, $6375=$ ILS 728; CIL 3, $5207=$ ILS 723; AE 1925, 72=I.L. Tun. 814; JUAN CRISOSTOMO, in ep. ad. Philipp. 4, 5; JUL.. Or. 1, 19 A; 1, 20 B; 2, 94 B; 2, 56; 2, 57 D; 2, 58 C; 2, 59 B; 1, 26 B; 1, 26 C; 2, 5 D; 1, 9 D; 2, 52 B; LIB. Or., 3, 283-323; 1; 59; MALALAS, 325, Bonn; MANSI 3, 144; COHEN 7, 402-35; OPT. 3, 1-3; Pan. Lat. 10, 36; PHILOST. HE 3, 1-12; RIC 7, 580; RIC 5, 268-300; SOCR. HE 2, 5-25; 1, 38; SOZ. HE 3, 2-20: 2, 34; 4, 1; SYMM. Rel. 8, 4; THEOD. HE 2, 3-8; 5, 21; Vita Olympiadis 2; ZON. Epit. 13,$5 ; 13,6$; ZOS. 2, 35-47.

(3) Este hecho ha llamado la atención de los historiadores modernos sin que hayan sido capaces de explicarlo satisfactoriamente. Cfr. JONES, $1973^{2}$, $1088 \mathrm{n}$. 1; BARNES, 1980, 160, pone de manifiesto que el período posterior a la muerte de Constantino es el más oscuro de la historia imperial romana. Sospecho que la falta de documentación sobre Constante pudiera ser responsabilidad de Constancio II, interesado probablemente en que el reinado de sus hermanos cayera en el olvido. 
Así pues, los datos de que disponemos al respecto son los siguientes. Eutropio y Zonaras afirman que a su muerte tenía 30 años, por lo cual debió nacer en el 320 (EUTR. Brev., 10, 9, 4; ZON. 13, 6). Por su parte, Aurelio Víctor y Malalas suponen que contaba con 27 años en el momento de su muerte; en consecuencia, habría nacido en el 323 (AUR. VICT. Epit., 41, 23; MALALAS, 325 Bonn). Por último, se conoce una moneda, por la cual posiblemente pudiera confirmarse en cierta medida una de estas dos fechas de nacimiento; de hecho, la consideración que ha merecido es que podría apoyar con más fuerza la datación del año 320 , por lo que a su muerte Constante contaría 30 años (SEECK, 1901, 948; SEECK, 1898, 39; COHEN, 1955, 402-35). Como es fácil observar la dificultad es enorme, pues no cuenta ninguna de las dos opciones con argumentos lo suficientemente sólidos como para obligar a un rechazo de la contraria. Esta opinión se refleja perfectamente en la valoración expuesta por la historiografía moderna, que no se ha resuelto de una forma clara en favor de una u otra propuesta. De este modo, advertimos que la actitud adoptada por J. Moreau de no decantarse por una fecha u otra (MOREAU, 1959, 179) ha sido retomada recientemente por A. H. M. Jones, J. R. Martindale y J. Morris en PLRE I, 220 n. 3. Sin embargo, la tesis tradicional representada por O. Seeck $(1898,38$; SEECK, 1901, 948) suponía que lo más probable era que el nacimiento de Constante hubiera tenido lugar en el año 323; asimismo, E. Stein $(1959,131)$ recogió esta postura y en la actualidad el defensor más representativo de esta tesis es T. D. Barnes (1981, 252; BARNES, 1982, 45; GAUDEMET, 1979, 255-6), quien advierte que a pesar de la dificultad que presenta esta cuestión, el año 323 cuenta con un apoyo más eficaz: «About his date of birth, the evidence diverges... However, a medallion which clearly celebrates his proclamation as Caesar depicts Constans as significantly younger than his brothers (RIC 7.580, Constantinople 67), so that 323 should be preferred over 320 for the date of his birth» (BARNES, 1982, 45). No obstante, ha surgido un sector historiográfico que se ha inclinado hacia el 320 como fecha más probable para el nacimiento de Constante; entre ellos ha destacado recientemente A. Chastagnol que se fundamenta en la edad que tenía en el momento de su nombramiento como César: «Le nombre des Césars fut notablement augmenté dans les dernières années: il passa à trois le 25 décembre 333 avec la proclamation du dernier fils de Fausta, Constant, alors âgé de 13 ans» (CHASTAGNOL, 1982, 128; cfr. PIGANIOL, 1972², 81; PALANQUE, 1938, 241).

Por todo lo expuesto se comprueba que no hay en modo alguno unanimidad respecto de esta cuestión, pero creo que es lógico intentar fijar, lo más aproximadamente posible, la fecha de nacimiento de Constante; por lo cual, se contará con un criterio preciso que permita determinar su capacidad de llevar el gobierno por sí mismo o si éste quedó en manos de otros en el momento en que se llevaron a cabo los repartos territoriales, tanto el del 335 como el del 337, a la muerte de su padre.

En mi opinión, hay un dato que puede representar un papel importante a la hora de dilucidar este oscuro problema. En efecto, disponemos del testimonio proporcionado en el año 321 por Pan. Lat. 10, 36: «Caesaribus nobilissimis habet eorumque fratribus» y que continúa con alabanzas hacia Crispo. La opinión expresada por E. Galletier con respecto de esta frase es clara: «Crispus et Constantin II et leurs frères Constance et Constant. Ces derniers sont si jeunes en 321 (étant nés en 317 et 320) que le panégyriste ne peut pas décemment leur souhaiter une proche accession à l'empire» (GALLETIER, 1952, 196 n. 1; PLRE I, 220 n. 3). En consecuencia, esta información significa específicamente un elogio para los césares existentes en el 321, que no son otros que Crispo y Constantino II (BARNES, 1982, 7-8; PLRE I, 233 n. 4; PLRE I, 233 n. 3), mientras que la referencia a sus hermanos no puede ser interpretada de otra forma más que como una referencia a Constancio y Constante, nacido hacía poco tiempo.

Con respecto del nombre de este emperador no se presenta dificultad alguna, puesto que está bien documentado por inscripciones y monedas que era conocido como 
Flavio Julio Constante (CIL II 6029=ILS 725; ILS 724; ILS 726; CIL III 2829; CIL III 6375 = ILS 728; CIL XI 5265 = ILS 705; BCH, 1946, 260 b; COHEN, 1955, 402-35). Por otra parte, era el cuarto o quinto hijo de Constantino el Grande, por lo cual debe entenderse que su madre era la segunda esposa del emperador cristiano: Flavia Máxima Fausta, hija de Maximiano Hercúleo. A pesar de que la mayor parte de la documentación, tanto inscripciones como textos literarios, coinciden en este punto (CIL II $6029=$ ILS 725; CIL III 5207 = ILS 723; LIB. Or. 59, 14; EUN. V. Soph. 6, 3, 8; JUL. Or. 1, 9 D; JUL. Or. 2, 52 B; LIB. Or. 59, 17; SOCR. 1, 38; SOCR. 2, 25; SOZ. 2, 34, 1; ZOS. 2, 35, 1; AUR. VICT., Caes. 41, 13; AUR. VICT., Epit. 41, 19), hay sin embargo un testimonio contrario, proporcionado por Zósimo (2, 39), según el cual Fl. Jul. Constante era hijo de una concubina de Constantino y no de Fausta, su esposa legítima. La opinión generalmente admitida en la historiografía moderna es que era hijo tanto de Constantino como de Fausta (SEECK, 1901, 948; SEECK, 1898, 42: MOREAU, 1959, 179; BARNES, 1982, 43-45: PLRE I, 220 n. 3), por lo cual se supone que Zósimo en dicho párrafo lo habría confundido con Crispo, quien efectivamente no era hijo de Fl. Max. Fausta, sino de Minervina, que probablemente fue la primera esposa de Constantino (PLRE I, 220 n. 3; BARNES, 1982, 44-45). Hay otra posibilidad, aunque sea difícil de demostrar, y es que Zósimo, en realidad, no estuviera confundiendo a los hijos de Constantino entre sí, sino que simplemente quisiera referirse tanto a los tres hijos de Constantino y Fausta, que fueron los que heredaron el Imperio, como a aquellos que no eran descendientes de Maximiano Hercúleo, aunque esta circunstancia no lo expresara con la claridad suficiente.

La educación recibida por este príncipe es igualmente una cuestión de gran interés, puesto que si llegáramos a conocerla estaríamos en disposición de comprender en qué medida había determinado su posterior programa de gobierno o había influido en su trayectoria política.

Hay constancia de que era una preocupación de Constantino la educación de sus hijos; en este sentido, debe recordarse que hace venir a Lactancio a la Galia encomendándole la instrucción de su hijo Crispo (JER. de vir. ill., 80; PLRE I, 233 n. 4). Pues bien, si es evidente que Constantino se ocupó de la formación de sus hijos, para nosotros también tendrá una gran significación, puesto que el conocimiento que tengamos de ello nos va a permitir calibrar los rasgos y caracteristicas predominantes que se manifiestan en un individuo que estaba destinado a dirigir y gobernar el Imperio, o al menos una parte de él; por lo tanto, llegar a saber qué aspectos educativos - políticos, económicos, culturales, religiosos, militares, etc.--, configuraron su instrucción interesa en gran medida. El mejor medio que se puede utilizar para estudiar esta cuestión consiste en analizar a los profesores encargados de impartirle su enseñanza. En primer lugar, es necesario citar el testimonio de Libanio, quien afirma que Constante fue educado por profesores famosos (LIB. Or. 3, 283); ello supondría que aquellos intelectuales mejor formados en los diversos campos del conocimiento fueron encargados de proporcionar a Constante una educación adecuada a su rango. Al menos, se conoce el nombre de uno de sus maestros, el de Emilio Magno Arborio (PLRE I, 220 n. 3; PLRE I, 98 n. 4; AUS. Prof. 16, 15; LIB. Or. 59, 31), con quien estudió latín; este individuo era un rhetor y abogado por lo cual no solamente le proporcionaría un conocimiento de la lengua latina y la oratoria, sino que también es de suponer que le ofrecería una formación técnica, desde el punto de vista literario, cultural y jurídico (4). En este aspecto, es interesante

(4) MARROU, $1975^{3}, 353$, opina que «tal era, en efecto, la gran originalidad de la enseñanza latina ofrecer a la ambición de los jóvenes la carrera judicial... en rigor de verdad, el juris prudens es un tipo original: es el hombre que sabe derecho, que conoce a fondo las leyes, las costumbres, las reglas de procedimiento...»; 348: «La enseñanza del rhetor latinus, como la del sophistés griego, tiene por objeto la maestría o el domino del arte oratoria, tal como lo asegura la técnica tradicional, el complejo sistema de reglas, procedimientos y hábitos progresivamente empleados por la escuela griega a partir de la generación de los sofistas»». 
destacar el hecho de que Constantino prefiriese mandar llamar a un individuo de la Galia para que se desplazase a la corte imperial de Constantinopla, a pesar de que en esta zona del Imperio existían escuelas y maestros famosos e importantes, lo que era una circunstancia que no escapaba a nadie (5). Los motivos que pudieran haber inducido a Constantino a solicitar la presencia de este personaje, de una familia curial del lejano territorio de la Galia -región más atrasada culturalmente que el Oriente- pudieran muy bien encontrarse en una conciencia por parte del emperador de sus propias raíces occidentales; aparte de que indudablemente conservaba lazos de unión con las gentes de esta zona; sabemos que Emilio Magno Arborio mantenía una amistad con los hermanos de Constantino (AUS. Prof. 17, 9-12; PLRE I, 98 n. 4). Así pues, se puede suponer que Constantino optó por dar a su hijo una imagen real o lo más exacta posible del Occidente imperial y una formación claramente occidental, con el fin de facilitarle posteriormente el gobierno de esta pars, a la que según parece lo había destinado. Aun cuando todas estas consideraciones no sean muy precisas tampoco hay rażón alguna, en mi opinión, que obligue a rechazarlas o no poder aceptarlas, al menos, en sus rasgos generales.

Por otra parte, llama la atención el que este maestro de Constante, Emilio Magno Arborio, no parece que estuviera adscrito de una forma clara a una determinada ideología religiosa (PLRE I, 98 n. 4); por ello, la afirmación que hace Eusebio de que los hijos de Constantino habían sido educados en el temor de Dios (EUS. Vita Const. 4, 51) no puede ser confirmada, por lo menos a partir del testimonio de este maestro. Con todo, esta cuestión la examinaré en otra ocasión, pues lo cierto es que, según parece, éste fue el único hijo de Constantino que se bautizó (OPT. 3, 3; ATHAN. Apol. ad Const. 7), hecho que posiblemente estuviera más en relación con las actitudes que Constante adoptara durante una etapa posterior de su vida que con el período de su educación en la corte oriental. Además Eusebio afirma también que Constante y sus hermanos eran educados en todas las virtudes imperiales. Ahora bien ¿en qué consisten las virtudes de un emperador según Eusebio? Es evidente que solamente es buen emperador aquel que practica las virtudes cristianas, puesto que, como ha indicado recientemente Barnes, Eusebio ha recogido la idea helenística de que la monarquía es el reflejo de un orden sobrenatural, el cual es lógicamente cristiano (BARNES, 1981, 253-271).

Hemos visto que para Constantino la educación de sus hijos era un tema que le preocupaba. Pues bien, de la misma forma se va a advertir que se interesa y ocupa de sus matrimonios. En concreto, en el caso de Constante, va a formalizar su compromiso con Olympia (PLRE I, 642 n. 1), la hija del PPO Fl. Ablabio (PLRE I, 3 n. 4), aunque el matrimonio sea aplazado porque ella no era núbil (AMM. 20, 11, 3; ATHAN. Hist. arr. 69; Vita Olympiadis 2 (ed. A. M. MARLINGREY, SC 13 bis, 1968, 408). Es evidente que interesa considerar las razones que movieron a Constantino para concertar este noviazgo; T. D. Barnes ha observado que «Constantine also arranged dynastic marriages for his sons... And although Constans, whose birth probably fell in 323, was too young for wedlock in his father's lifetime, he was betrothed to Olympias, the daughter of Ablabius. These matches clearly reflects Constantine's intention to leave his sons under the tutelage of advisers he had himself chosen» (BARNES, 1981, 252). Estoy de acuerdo con esta opinión, pues parece claro que Constantino pretendía que sus hijos se encontrasen bajo la tutela de aquellos consejeros que él previamente hubiese seleccionado; por lo tanto, ello bien puede significar que un objetivo de tales uniones consistía en

(5) MARROU, $1975^{3}, 372$, opina que «...en el siglo IV tales escuelas aparecen más o menos por todas partes... se hallan atestiguadas en la Galia por Ausonio en las ciudades de Lyón y Besançón y probablemente en Tolosa; por San Agustín en Cartago y Milán; en Oriente, por Libanio, en Constantinopla, Nicomedia y Nicea y también en Antioquía. Puede admitirse que para aquel entonces toda ciudad, por escasa que fuera su importancia, había tomado a su cargo el sostén de un profesor o varios». 
fortalecer o afianzar los lazos con ciertos sectores de poder. Ahora bien, en el caso de Constante, ¿por qué deseaba el emperador cristiano llegar a una relación tan estrecha, como es la de parentesco, con Fl. Ablabio, un individuo de baja extracción social (LIB. Or.. 42, 23; EUN. V. Soph. 6, 3, 1; PLRE I, 3 n. 4), a quien con esta medida elevaba sociopolíticamente de forma tan extraordinaria? (6). Es de suponer que alguna ventaja presentaría para la familia imperial dicho matrimonio, pero ¿qué tipo de ventaja?; no debemos olvidar que Constante era enviado a Occidente y se le encargaba el mando, cuidado o vigilancia de territorios occidentales; regiones donde no parece que Fl. Ablabio tuviera ningún poder o influencia, puesto que él, que era originario de Creta, prácticamente desarrolló toda su carrera en Oriente (7); además, sus posesiones: tierras y una casa, se encontraban igualmente en la pars orientis (EUN. V. Soph. 6, 3, 10; SYN. Ep. 61). Así pues, creo que estas razones parecen confirmar la idea de que sus contactos y relaciones con la zona occidental no debían revestir importancia alguna. Por otra parte, hay un hecho que debe tenerse en cuenta y es que Fl. Ablabio había sido nombrado PPO de Constancio (8); en consecuencia, se le había concedido un área muy amplia de influencia, puesto que no sólo estaba en relación, eminentemente favorable, con respecto de Constante, sino que también se le había situado muy cercano de Constancio, el próximo emperador de Oriente. Por lo tanto, pudiera ser que con tales medidas Constantino pensara que había asegurado a su hijo menor un apoyo seguro y eficaz por parte de Constancio y de las fuerzas orientales gracias a la presencia y presión de Fl. Ablabio, de tal manera que estarían neutralizadas posibles amenazas, quizá no muy bien precisadas, aunque sí advertidas con anterioridad. Al respecto, se debe recordar un dato proporcionado por Aurelio Víctor (Caes. 41,14), según el cual la noche que siguió al nombramiento de Constante como césar hizo su aparición una aurora boreal; dicha circunstancia fue interpretada como de mal agüero para su reinado. En definitiva, si se puede aceptar esta explicación, es decir, que en efecto existe una relación entre estos hechos, quizá pudiera entenderse mejor el compromiso establecido entre Constante y Olympia. Otro aspecto de esta cuestión que debe considerarse se refiere a la actitud de Constancio. En efecto, se advierte que los lazos de unión previstos por Constancio entre su propia dinastía y Fl. Ablabio no eran del agrado de Constancio II, quien no estaba dispuesto a hacerse cargo en su totalidad de los compromisos o proyectos de su padre y esto lo demostró de inmediato, ya que tras morir su padre, procedió rápidamente a deponer a su PPO, Fl. Ablabio, y ordenó su ejecución al año siguiente, en el 338 (EUN. V. Soph. 6, 3; ZOS. 2, 40, 3; JER. Chron. s. a. 338). Todo ello corrobora la hipótesis de que Constantino pretendía que Constante conservara apoyos políticos en el Oriente, cerca de Constancio, lo cual se lograría a través de Fl. Ablabio.

El nombramiento de Flavio Julio Constante como césar tuvo lugar en una fecha muy temprana, pues tanto si tenía 13 ó 10 años - en los casos de que hubiera nacido en el 320 o en el 323-, lo cierto es que dicho nombramiento sucedió en el año 333, más exactamente el 25 de diciembre del 333 (AUR. VICT. Caes. 41, 14; Chron. Min. 1, 232235; EUS. Laud. 3; EUS. V. Const. 4, 40; LIB. Or. 3, 283). La elección de esta fecha concreta es muy significativa, con claras implicaciones ideológicas, lo cual ya fue advertido por A. Piganiol, quien destacó el hecho de que el 25 de diciembre era el día en el que tradicionalmente se conmemoraba el nacimiento del sol (PIGANIOL, 1972 ${ }^{2}, 61$ ),

(6) CHASTAGNOL, 1968, 334, opina que Fl. Ablabio era un «hombre nuevo» y que su ascensión al clarisimado había sido por vía de adlectio, seguramente antes de ser nombrado PPO. sul.

(7) Fue oficial del gobernador de Creta, senador de Constantinopla, Vicario de Asiana, PPO y cón-

(8) Hecho que está ampliamente documentado: CTh 11, 27, 1; 16, 2, 6; 13, 5, 5; 16, 8, 2; 5, 9, 1; P. Oxy. 990; P. Lond. 3, p. 232, n. 978; ATHAN. Fest. Ep. 4; CTh 3, 16, 1; 7, 22, 5; ILT 814; EUN. V. Soph. 6, 2, 10-12; AMM. 20, 2, 3; ZOS. 2, 40; PALL. Hist. Laus. 56; cfr. PLRE I, 3 n. 4; BARNES, 1982. 132. 
por lo cual la nueva ideología dominante trataba de imponer, a partir de ahora, la identificación de este día con el nacimiento de Cristo (GARCIA IGLESIAS, 1979, 367; HERZ, 1975; LIEBESCHUETZ, 1979); en este sentido, el acto solemne que representa la proclamación de un nuevo césar podría muy bien ser considerado y mostrado como una especie de «revelación», que en este caso daba a conocer un nuevo defensor de la fe cristiana.

Tras su nombramiento como césar, Constante permaneció aún unos años en Constantinopla, junto a su padre, según el testimonio de Libanio (Or. 59, 42; BARNES, 1982,86 ), hasta el año 335; es probable que se adoptara esta decisión en espera de que el joven hijo de Constantino adquiriese una edad más adecuada para desempeñar ciertas tareas de gobierno.

En efecto, ya en el 335 Constantino procede a llevar a cabo un reparto de áreas de gobierno entre sus herederos, hijos y sobrinos, con adjudicaciones concretas de territorios a cada uno de ellos, tal y como testimonian diversos documentos (9). De tal forma que la opinión generalmente admitida es que a Constantino le correspondió Galia, Hispania y Britania; a Constancio, Asia y Oriente; a Constante, Italia, Africa e Ilírico; a Dalmacio, la ripa Gothica (Tracia y Macedonia), y a Anibaliano le entregó Armenia (PIGANIOL, 1972² , 60-1; STEIN, 1959, 131; JONES, 1973² I, 85; BURCKHARDT, 1982, 323; MOREAU, 1959, 179; SEECK, 1901, 948; PALANQUE, 1944, 47; BARNES, 1981, 252; BARNES, 1982, 198). No obstante, es de interés examinar, en el caso de que sea posible, si este reparto territorial previsto por Constantino se materializa en la realidad con la entrega de la soberanía de tales gobiernos y si, en efecto, cada uno de los césares y en concreto Constante, acuden a hacerse cargo de las responsabilidades que se les habían encomendado, mediante su establecimiento en el territorio. En el caso de que no se diera dicha circunstancia, es decir, que no se hubieran dirigido ni tampoco hubieran residido en estas regiones, se podría concluir que el reparto gubernamental del 335 era únicamente un estudio en el plano teórico y que las fuentes documentales habían informado exclusivamente sobre un proyecto político de Constantino como era el de la herencia imperial. Por otra parte, es necesario observar si la relación de cada uno de los césares con respecto de sus territorios era diferente: el que unos hubieran acudido a ellos y otros no, etc.; ello supondría que la realidad consistía en que se trataba de una prefiguración de lo que exactamente se pretendía fueran los gobiernos imperiales posteriores a Constantino.

En primer lugar, se dispone de un dato y es que a la muerte de Constantino, como se verá, ninguno de sus presuntos herederos se encontraba en Constantinopla; este hecho puede interpretarse precisamente como una muestra de que residían en las regiones que el monarca cristiano les había encomendado en el 335. En efecto, si nos detenemos en comprobar documentalmente el lugar de residencia de cada uno de los césares observamos que Constantino II se encuentra en Tréveris (ATHAN., Apol. Sec. 87, 4); Constancio reside en Antioquía (ZON. 13, 4); Dalmacio estaba en Naissus (CJ 5, 17, 7); mientras que Anibaliano se había establecido en Cesarea de Capadocia (CHRON. Pasch. 531, Bonn; PIGANIOL, 1972 2 , 61-2); con respecto de Constante, o bien se admite que «nous ignorons où il réside» (PIGANIOL, 1972², 81-2), o bien se supone que en ese momento se encontraba en Milán (BARNES, 1982, 86); esta alternativa no es posible resolverla en la actualidad debido al estado de la documentación que silencia totalmente la situación del hijo menor de Constantino en este período de tiempo (10); sin

(9) BARNES, 1982, 198, opina que «Eusebius, VC 4. 51, 1, deliberately confusing the divisions of 335 and 337». Anon. Val. 35; AUR. VICT. Epit. 41, 20; EUS. V. Const. 4, 51, 1.

(10) BARNES, 1980, 160, opina que «The period after the death of Constantine on 22 May 337 is among the most obscure in Roman imperial history". 
embargo, una prueba de que probablemente su estancia la había fijado en Milán o en definitiva en Italia, podía proceder de la evidencia prosopográfica proporcionada por L. Papio Pacatiano (PLRE I, 656 n. 2), quien fue PPO desde el 332 al 337 y que, sin duda, se encontraba en Italia en el 335 (CTh. 8, 9, 1); por lo cual, este PPO adscrito a Constante le acompañaba mientras que el césar residía en la Península.

En definitiva, se puede concluir que la documentación apoya la idea de que Constante - como el resto de los césares-, ya en el 337, se había hecho cargo del gobierno de los territorios que Constantino le había adjudicado en el reparto imperial del 335 . Así pues, si esto se acepta, se advierte que la idea tradicional expresada por A. Piganiol: «Les jalousies entre les fils et les neveux de Constantin expliquent que le partage préparé per Constantin en 335 ne fut pas réalisé» (PIGANIOL, 1972², 82; STEIN, 1959, 131, 484) no puede ser acertada, puesto que si bien el conflicto que surge en el 337 tras la muerte del emperador se debe a distintos condicionamientos, entre los que efectivamente hay que contar con los de las competencias y tensiones entre los diferentes herederos, lo que no creo que sea correcto es considerar que los tumultos tras el 22 de mayo del 337 son una prueba de que no se había puesto en práctica el reparto del 335. En mi opinión, sucede lo contrario, creo que se habían adjudicado los gobiernos territoriales en el 335 a cada uno de los césares; por lo que naturalmente cada uno de ellos se había establecido en su región y se había hecho cargo de su administración. En consecuencia, era precisamente esta circunstancia lo que suponía una grave amenaza para el objetivo de lograr la hegemonía estrictamente constantiniana en todo el Imperio y lo que provocó la masacre del 337.

Como se sabe, la muerte de Constantino tiene lugar el 22 de mayo del 337. Este acontecimiento no parece provocar en el Imperio ningún vacío de poder y en el conjunto territorial del Estado no se manifiesta ningún signo de inestabilidad. Ello viene a confirmar lo que anteriormente se había supuesto: que cada uno de los césares estaba ya instalado y en el ejercicio del gobierno de las regiones que les habían correspondido en el reparto del 335. Por lo tanto, la continuidad en el mando estaba asegurada; además, es muy probable que la falta de alborotos se debiese a la idea que pudieran tener los ciudadanos romanos de que la sucesión hereditaria había sido llevada a cabo antes de que se produjera el fallecimiento de Constantino; en consecuencia, para ellos la suprema autoridad de éste como único emperador del Imperio, durante esta etapa de tiempo, bien pudo ser exclusivamente un hecho teórico, puesto que la realidad inmediata era que el gobierno de cada uno de los territorios que componían el Estado se encontraba en manos de sus hijos y sobrinos. Es muy posible que esta hipótesis tenga más fuerza respecto de Occidente debido a la especial circunstancia que suponía su lejanía del poder central. Por todo ello, las distintas fuerzas sociopolíticas debían considerar que no se habia producido ruptura en la continuidad del poder imperial en razón a que un césar, acompañado por un equipo de gobierno, hacía tiempo que estaba instalado en estos territorios y dirigía su administración con libertad. En definitiva, la desaparición del lejano Jefe del Estado no podía provocar un trastorno importante en la relación de fuerzas imperantes.

Ahora bien, la muerte de Constantino sí provocó una conmoción que se manifestó en la más elevada área del poder: entre los herederos. En efecto, la crisis estalla en el mismo aparato gubernamental y creo que no participan de ella el resto de las fuerzas sociopolíticas.

Se hace necesaria una explicación más detenida de este fenómeno. En primer lugar, considero conveniente tomar este conflicto como una crisis «sostenida» por los hijos de Constantino, puesto que ellos son los responsables del mantenimiento durante los meses posteriores al 22 de mayo de una situación cuando menos extraña y sin lugar a dudas confusa, al no proclamarse augustos; esto significaba que el Estado romano se encontraba acéfalo, aunque se continuara legislando en nombre del emperador muerto (CTh 
13, 4, 2; PIGANIOL, $1972^{2}$, 82: VOGLER, 1979, $16 \mathrm{n}$. 14). El sentido de este estado de cosas puede entenderse ahora con cierta claridad. Los hijos de Constantino perseguían un objetivo: ser ellos los únicos dueños de la inmensa herencia legada por su padre; para ello, precisaban disponer de cierto tiempo, un compás de espera en el que seguramente organizarían sus fuerzas y consolidarían sus posiciones. Es evidente que para lograr la total hegemonía y un completo dominio del Estado no había más alternativa que la de eliminar a sus rivales, los parientes que el emperador fallecido había designado también como legítimos herederos del Imperio. Por lo tanto, mi opinión es que los trágicos sucesos del 337 hay que entenderlos y explicarlos como una consecuencia de los conflictos y tensiones existentes en el mismo aparato gubernamental.

No obstante, se ha señalado en repetidas ocasiones que el inmediato responsable de estos acontecimientos fue el ejército (EUS. Vita Const. 4, 69, 2; ZOS. 2, 39-40; JUL. Ep. ad Atha. 270 C; ATHAN. Hist. Arian. 19; JUL. Pan. 5, 3; BURCKHARDT, 1982, 326-7; SEECK, 1901, 948; STEIN, 1959, 131; PIGANIOL, 1972², 83; OLIVETTI, 1915, 67-79; BARNES, 1981, 262; CHASTAGNOL, 1969, 24; CHASTAGNOL, 1982, 131-2; REMONDON, 1973, 75). En efecto, se ha sostenido que esta institución era partidaria de que la sucesión estuviese reducida a la línea dinástica directa: los hijos. Por esta razón debió tramar y llevar a cabo la masacre de gran parte de la familia constantiniana; igualmente, se indica que Constancio II participó de dicha responsabilidad, puesto que no intervino para evitar los asesinatos.

No creo que esta explicación sea la acertada, puesto que hace únicamente al ejército, como institución, directo responsable de esta acción y según parece se trata del ejército de Constancio, puesto que él es el único a quien se le menciona en relación con estos hechos. La objeción inmediata que se me ocurre es que si esto hubiera sido así lo lógico sería que toda la acción hubiera ido dirigida a consolidar exclusivamente el poder de Constancio, quien no desearía compartirlo con nadie, por lo que también hubieran sido eliminados sus hermanos. Sin embargo, nada de eso ocurrió; lo que hace que me reafirme en la idea de que la masacre no fue un acto urdido y programado por el ejército, sino que fue una actuación realizada por los soldados de Constancio, quien sin lugar a dudas estaba en connivencia con Constantino y Constante. En definitiva, el complot fue obra de los hijos de Constantino el Grande.

Por otra parte, se hace preciso tener en cuenta la explicación que sobre estos acontecimientos ha dado recientemente T. D. Barnes $(1981,262)$. El interpreta, por las palabras de Eutropio: sinente potius quam iubente (EUTR. Brev. 10, 9, 1), que la voluntad imperial no intervino de forma alguna y que estos hechos fueron causados, dirigidos y realizados por dos generales del ejército: Fl. Urso (PLRE I, 984 n. 4; BARNES, 1974, 226-7) y Fl. Polemio (PLRE I, 710 n. 4), que luego en el 338 fueron designados cónsules, lo que provocó el desplazamiento del cónsul nombrado para ese año con anterioridad, Q. Fl. Egnatio Lolliano Mavortio (PLRE I, 512 n. 5).

De hecho T. D. Barnes ofrece una visión simplista de este problema, sin que en él se den implicaciones de mayor envergadura. Por mi parte, opino que en las palabras de Eutropio debe verse precisamente una acusación contra el poder imperial, ya que si afirma que éste ha permitido que se realice la acción, ello es como decir que hay un conocimiento y por lo tanto una participación; además, conocemos un dato que merece ser recordado, y es que los dos oficiales del ejército, autores de los crímenes, van a ser recompensados, y es evidente que siempre se premia a aquellos que han cumplido con las órdenes recibidas. Así pues, aunque no comparto la hipótesis de T. D. Barnes en cuanto a que libera de responsabilidad a los hijos de Constantino, lo cierto es que su explicación no afecta negativamente a una parte de mi suposición, la de que la actuación de Fl. Urso y Fl. Polemio no implica necesariamente que éstos fueran las cabezas visibles de una conjura militar. En efecto, sostengo que estos generales no pueden ser considerados como los representantes, ni tan siquiera, de una facción del ejército que pusiese de ma- 
nifiesto, con la masacre de los familiares de Constantino, un proyecto político o una alternativa de poder; ellos únicamente han realizado un acto, instigado y favorablemente consentido por una autoridad superior, su césar (11).

Sea como sea, lo cierto es que la desaparición de gran parte de los miembros colaterales de la familia constantiniana significó que los hijos de Constantino contasen con una total libertad para llevar a cabo un reparto territorial según sus propios intereses. En primer lugar, se constituyen como un nuevo colegio imperial, pues el 9 de septiembre los tres hermanos se proclaman augustos, lo cual es confirmado por el Senado de Roma (EUS. Vita Const. 4, 68; CHRON. MIN. I, 235; PALANQUE, 1944, 55; DEMOUGEOT, 1979, 243; BARNES, 1981, 252). A continuación, celebran una entrevista en Viminacium (12), donde acuerdan una nueva distribución del Imperio, la cual presenta bastantes dificultades para ser establecida en la actualidad a pesar de que se fundamenta y reposa en la del 335 (13). En efecto, está claro que cada uno de los hermanos conserva el gobierno de las regiones que su padre les había asignado. Así pues, Constantino mantiene bajo su autoridad Galia, Britania e Hispania, sin que haya noticias de que aumente su área de gobierno; Constancio domina ahora todo Oriente y toda Asia Menor; mientras que Constante tiene en su poder Panonia, Italia, Africa y se hace con Macedonia. Por ello, el problema es muy concreto, pues sólo es conflictiva la adscripción de la diócesis de Tracia debido a la ambigüedad que presentan los textos antiguos (PHILOST. HE 3, 1. ${ }^{\text {; }}$ ZON. 13, 5; ZOS. 2, 39; AUR. VICT., Epit. 41, 20), los cuales permiten dos interpretaciones opuestas: la que dio E. Stein, seguido por la mayoría de los historiadores, que consiste en considerar que Tracia correspondió a Constancio (STEIN, 1959, 132; JONES, $1973^{2}$, 112-115; PETIT, 1974, 599; BARNES, 1981, 252, 262; BARNES, 1982, 198-199), o bien la propuesta por O. Seeck, quien supuso que fue entregada a Constante, quien la cedió a Constancio en el 339, una vez que inició la guerra contra su hermano mayor Constantino (SEECK, 1895-1921, IV, 42, 46, 397, 400; SEECK, 1901, 949; PALANQUE, 1944, 57 n. 4). Recientemente C. Vogler, en su fundamental estudio sobre Constancio II, ha tratado igualmente el tema; pretendió resolver la cuestión por la información que diera el Código de Teodosio y comprobó que ello no ofrecía ninguna posibilidad, por lo que concluyó que ignoramos cuál es la atribución de Constantinopla y Tracia hasta el año 340 (VOGLER, 1979, 16 n. 14). Por mi parte, he considerado que sería necesario recurrir a otro tipo de documentación: la prosopográfica, con la suposición de que el conocimiento que obtuviera de los magistrados de esta región, en el trienio 337-340, me permitiría determinar a qué emperador había correspondido. Desgraciadamente la evidencia prosopográfica, en esta ocasión es muy escasa (PLRE I, Fasti, 1085 ss.), ya que solamente tenemos los nombres de dos individuos que desempeñaron una función del Estado en esta región durante esas fechas. En primer lugar, tenemos a

(11) BARNES, 1981, 398 n. 17, reconoce que no sabemos prácticamente nada de Fl. Urso y Fl. Polemio en fechas anteriores y posteriores al 337-338. Esto puede interpretarse en el sentido de que no eran personajes importantes y que no desempeñaban ningún papel de valor en la política del gobierno imperial; lo cierto es que su papel se había reducido exclusivamente al jugado en favor de los intereses de su césar.

(12) La fecha de esta reunión no se ha podido determinar con exactitud; pudo realizarse en el mismo año 337 o bien en el 338. ATHAN. Apol. Sec. 87, 4; Frag. Vat. 35; JUL. Or. 1, 19 A; LIB. Or. 3 , 297 ; cfr. CHASTAGNOL, 1982, 132; SAYAS, 1981, 29; MOREAU, 1959, 179; SEECK, 1901, 949; JONES, 19732 , 112-115; BARNES, 1981, 252, 262; BARNES, 1980, 160; PIGANIOL, 1972 2, 82; DEMOUGEOT, 1979, 243.

(13) Hay diversos testimonios documentales que dan lugar a diferentes interpretaciones en la actualidad. Anon. Val. 35; ATHAN. Apol. Sec. 87, 4; Chron. Min. 1, 235; AUR. VICT. Epit. 41, 20; EUS. V. Const. 4, 51; 4, 68; EUTRO. Brev. 10, 6; JUL. Or. 1, 19 A, 20 B; LIB. Or. 3, 297; Pass. S. Artemii $8=$ PHILOST. HE 3, 1; ZON. 13, 5, 1; ZOS. 2, 39; 2, 41; BARNES, 1982, 198-199; BARNES, 1981, 252; JONES, 1973 2, 112-115; SEECK, 1901, 949; DEMOUGEOT, 1979, 243; BARNES, 1980, 160; VOGLER, 1979, 14 ss.; CHASTAGNOL, 1982, 132; SAYAS, 1981, 29; STEIN, 1959, 131-2; PIGANIOL, 1972 2, 83; PETIT, 1974, 599. 
Fl. Palladio (PLRE I, 661 n. 16 y n. 17; JONES, 1974, 270; GARRIDO, 1984). Si se pudiera determinar fehacientemente el año exacto dentro del período en cuestión, en el que ocupó su cargo, entonces el hecho de que la carrera administrativa de Fl. Palladio muestra que debió a todas luces ser un hombre de confianza de Constancio (GARRIDO, 1984), apoyaría la suposición de que fue este emperador quien administró dicha diócesis. Con todo, como no es posible establecer exactamente dicho año, carece de fundamento concluir la posible administración de este territorio por parte de Constancio.

En segundo lugar, conocemos a Sappo (PLRE I, 803), quien fue dux de Scytia, igualmente entre el 337 y el 340 . Por ello, los autores de la Prosopografía han supuesto que estuvo bajo la autoridad de Constantino II; sin embargo, dicha hipótesis es totalmente gratuita, porque la inscripción por la que se le conoce no ofrece ningún dato que la apoye (CIL III, 12483 = ILS 724). Al contrario, creo que en ella se ofrece una información, respecto de un hecho militar, que puede interpretarse en favor de Constante. En el caso de que esta interpretación fuera acertada se concluiría que Sappo fue un $d u x$ nombrado por Constante para que cumpliera su función en un territorio que estaba bajo su autoridad.

En efecto, en dicho epígrafe se da el título de Sarmaticus a Constancio y a Constante; ello significa que ambos llevaron a cabo una expedición victoriosa contra los Sármatas en el año 337 (BARNES, 1981, 262; BARNES, 1980, 162; BARNES, 1976, 154; THOMPSON, 1956, 372-381). Por lo tanto, deduzco como probable el que fuera Constante el responsable de haber organizado tal campaña en una gran zona incluida en su área de poder y Constancio acudió únicamente como fuerza de apoyo.

Las razones en las que baso mi suposición son tanto la premura con la que se realiza esta acción militar, como la misma participación de Constante en ella.

En mi opinión estos dos hechos se entienden si se admite que es Constante quien domina la Tracia, pues en tal caso es comprensible que desee iniciar rápidamente las hostilidades contra un peligro que amenaza o puede amenazar su territorio; lógicamente su participación se debe a que interviene en defensa de una zona que está directamente bajo su autoridad. En este mismo supuesto no es difícil explicar la participación de Constancio, porque sabemos que le interesaba en gran medida la salvaguarda de esta región de la Tracia, territorio sobre el que pretende establecer su dominio, como se demuestra años después.

Por otra parte, si aceptamos la hipótesis de que Constancio dominaba la Tracia en estas fechas, resulta difícil de explicar la rapidez con la que se comenzó la expedición sármata, pues considero como más probable que hubiese esperado a que finalizara la campaña proyectada en Oriente, la cual llevó a cabo en el 338 (BARNES, 1980, 162; BARNES, 1976, 154). Además, si la primera acción bélica hubiera obedecido a motivos muy graves, Constancio no habría emprendido una nueva campaña dejando a otro emperador la responsabilidad de dirigir la defensa de su propio territorio. Ahora bien, si la acción en Tracia no se debía a un peligro muy serio, sino únicamente consistía en una demostración de fuerza o en un acto de represalia, en tal caso no hubiera sido necesaria la participación de las fuerzas de Constante.

En definitiva, sostengo que la actuación conjunta de los dos emperadores en la expedición de Tracia contra los sármatas en el 337, se explica más fácilmente si se reconoce que esta diócesis se encuentra en esas fechas bajo la autoridad de Constante.

En conclusión, creo que, en el estado actual de nuestros conocimientos, es preferible mantener la teoría de que fue Constante quien obtuvo el dominio de la diócesis de Tracia en el 337.

Incluido en el límite cronológico del trienio 337-340 hay un tema que ha resultado siempre polémico en la historiografía moderna. Me refiero a la supuesta supremacía de Constantino II en el colegio imperial formado con sus hermanos y en concreto a la tute- 
la ejercida por éste sobre el menor Constante, al cual incluso se le ha llegado a calificar como un augusto «sans terre» (PALANQUE, 1944, 58). La documentación que ha servido de base para la formulación de esta hipótesis es variada: inscripciones, leyes y textos literarios (CTh 12, 1, 28; ZOS. 2, 39, 2; LIB. Or. 59, 46; AUR. VICT. Epit. 41, 20; ANON. VAL. 35; CIL II $474=$ ILS 130; AE 1927, 165; MITFORD, 1939, 187).

Por mi parte pretendo demostrar que la supuesta preeminencia de Constantino II se dio en efecto; pero únicamente con carácter honorífico, sin que en la realidad aquélla se correspondiera con una tutela o una soberanía sobre alguno de sus hermanos o respecto de sus territorios.

El primero que expuso la teoría de que el territorio occidental no se había dividido entre Constantino II y Constante fue O. Seeck, quien se basó en los testimonios de Zósimo, Anónimo Valesiano y Aurelio Víctor, y supuso que el primogénito de Constantino el Grande había ejercido una tutela sobre su hermano menor (SEECK, 1895-1921, IV, 41-2, 397).

La crítica a la teoría de O. Seeck fue presentada por J. R. Palanque, quien afirmó rotundamente que «cette solution ne résiste pas à l'examen». Este investigador nos obliga a recordar que los textos mencionados se refieren a la distribución territorial que se realizó en vida de Constantino entre sus hijos y sobrinos; por lo cual, dichos documentos no tienen relación con los acontecimientos sucedidos, ni con los repartos nuevamente practicados, después de la muerte de Constantino, y una vez que sus hijos han eliminado a sus parientes (PALANQUE, 1944, 56-58). Así pues, la conclusión propuesta por el sabio francés es que en el 9 de septiembre del 337 no se llevó a cabo un reparto territorial más que entre dos hermanos: Constantino II y Constancio II, mientras que Constante quedaría con el título de augusto, pero «sans terre»; por lo cual, la posterior rebelión de éste tenía por objetivo la usurpación de la soberanía de Constantino II en todo Occidente (PALANQUE, 1944, 58).

En mi opinión, considero muy acertada la crítica de J. R. Palanque a las teorías de O. Seeck, pero su deducción posterior me parece carente de fundamento, debido a que no cuenta realmente con ningún apoyo documental. Además, como ya ha señalado $C$. Vogler, en el caso de que se admita la supremacía de Constantino II sobre Constante se hace preciso responder satisfactoriamente a una interrogante: «... a-t-on le droit de supposer que Constant ait été privé des prérogatives afferentes à son titre d'Auguste?» (VOGLER, 1979, 16 n. 14); por mi parte, lo creo muy improbable.

En definitiva, no hay base literaria alguna que permita sostener que Constante estaba bajo la autoridad de su hermano mayor Constantino II y que, por lo tanto, no ejercía sus legítimos poderes de augusto sobre los territorios que le habían correspondido. Ahora bien, lo que sí se puede aceptar es que en el colegio imperial se le reconocía a Constantino II una supremacía honorífica por ser el mayor, y ello se reflejaba en sus títulos y en la preeminencia del lugar que ocupaba en los monumentos.

Por otra parte, difiero de la suposición de J. R. Palanque de que la causa de la guerra entre ambos hermanos fue debida al intento del menor de usurpar el poder en Occidente. Considero por el contrario que la provocación procedió de Constantino II, quien pretendía hacer efectiva una supremacía que sólo era reconocida en el plano honorífico.

Las inscripciones son otro tipo de documentación utilizado en favor de la tesis de la tutela de Constantino II sobre Constante. En concreto se trata de dos epígrafes: uno procedente de Mérida y otro de Chipre (AE, 1927, 165; CHASTAGNOL, 1976, 260 ss.; MITFORD, 1939, 187; PIGANIOL, 1972² 83 n. 3); en ambos se puede leer el título de maximus atribuido a Constantino II; en cambio, Constancio II y Constante llevan únicamente el título de victor. Estas diferencias en la titulación han hecho pensar a prestigiosos historiadores que la tesis tradicional era acertada.

No obstante, creo que tampoco este testimonio apoya dicha interpretación. Al con- 
trario, sirve más bien para demostrar la suposición que he propuesto más arriba: a Constantino II le correspondió únicamente una supremacía honorífica.

En efecto, es preferible pensar que a Constantino II, por el hecho de ser el augusto de mayor edad, le corresponde un título de mayor honor: el de maximus; pero ello no implica necesariamente que tuviera más prerrogativas de poder o autoridad que sus hermanos y desde luego no contaba con ninguna soberanía sobre los territorios gobernados por los otros emperadores.

Por otra parte, aun en el caso de que fuera cierto que tenía alguna supremacía, debo luego entender que ésta debía ejercerla por igual sobre los demás augustos y no sobre uno solo de ellos; además, su tutoría se extendería sobre todos los territorios y no estaría limitada a una parte de ellos. Sin embargo, nadie duda que Constantino II no contaba con ninguna autoridad sobre Constancio II y Oriente; por lo tanto, si el título de maximus no presupone que Constantino dominara a Constancio, que sólo lleva el título de victor, habrá que considerar que el mismo tipo de relación se habría establecido entre Constantino y Constante, pues se repiten las mismas condiciones.

Las mismas objeciones se le pueden presentar a otro argumento de O. Seeck, fundamentado en un medallón del año 338, en el que figuran los tres hermanos, pero únicamente Constantino II lleva el nimbo (SEECK, 1894, 44; PIGANIOL, 1972², 83 n. 3; TOYNBEE, 1944, 199; BARNES, 1981,399 n. 25; BARNES, 1982, 45; VOGLER, $1979,16 \mathrm{n} .14)$. Creo que precisamente ello se debe a que los otros dos augustos reconocen en el mayor una preeminencia de tipo honorífico. Ahora bien, en el caso de que aceptáramos que ello es la representación de una tutoría sobre Constante, entonces necesariamente tiene que representar también una tutoría sobre Constancio; como tal interpretación no parece que se pueda mantener de momento, tampoco se debe admitir en el otro caso. Además, no se debe olvidar la crítica de $\mathbf{M}$. Thirion, quien asegura que la tesis de $\mathrm{O}$. Seeck se basa en una apreciación errónea de las monedas conmemorativas que fueron emitidas después del 9 de septiembre del 337 (THIRION, 1965, 8 ss.).

Por último, la tesis tradicional ha presentado también como dato a su favor la ley CTh 12, 1, 27 enviada por Constantino II desde Tréveris al procónsul de Africa Aurelio Celsino (PLRE I, 192 n. 4; GARRIDO, 1983, 924, 1003), el 8 de enero del 339; por ella se ha afirmado que el acto de legislar le correspondía exclusivamente a Constantino II, porque él era el único emperador de Occidente (BARNES, 1981, 399 n. 1; BARNES, 1982, 200).

En mi opinión, esta interpretación debe ser reconsiderada, pues hay una explicación más satisfactoria de por qué Constantino II dio una ley para un territorio incluido en la jurisdicción de Constante. Puede plantearse que muy posiblemente en enero del 339 se encontraba aún Constante en la campaña que había emprendido contra los sármatas en el 337 ó 338 (ILS 724; ZON. 13, 5, 9; BARNES, 1980, 164; BARNES, 1982, 86; SEECK, 1919, 187; cf. supra); por lo tanto, durante su ausencia, Constantino II se habría visto obligado a tomar una decisión de gobierno que se hacía precisa.

Por otra parte, J. R. Palanque propuso, en su fundamental trabajo del año 1944, un nuevo método por el cual se podría comprobar si un emperador ejercía una verdadera soberanía. Según él era necesario, en primer lugar, que se hubieran conservado en el Código constituciones indudablemente promulgadas por dicho augusto; una segunda condición consistía en que el emperador tuviera ministros: un magister officiorum, un quaestor palatii, un comes rei privatae y un comes largitionum; por último, que hubiera nombrado magistrados para el desempeño de ciertas funciones administrativas. En definitiva, él concluía que «l'existence d'une cour ou d'une législation indépendantes est donc nécessaire pour nous permettre en toute rigueur d'affirmer la souveraineté d'un Auguste du Bas-Empire») (PALANQUE, 1944, 49).

En mi opinión, es preciso llevar a cabo una comprobación de que las condiciones exigidas por J. R. PALANQUE se dan en el gobierno de Constante durante el trienio 
337-340; de forma que se confirme la idea de que el hijo menor de Constantino el Grande ejerció su soberanía desde el primer momento, al igual que sus hermanos.

Con respecto a la legislación hay que advertir que nos estamos moviendo en un período de tiempo muy corto, motivo por el cual no debe extrañar que el volumen de leyes conservadas no sea muy elevado; además, es útil recordar la observación de C. Vogler en cuanto a que las constituciones recogidas en el Código presentan frecuentemente una gran dificultad a la hora de intentar establecer su procedencia; especialmente ello afecta a la legislación anterior al 350; en realidad, C. Vogler sospecha que la atribución de las leyes del trienio 337-340 quedará como un problema insoluble (VOGLER, 1979, 14-16).

No obstante, destacan algunas leyes inscritas en tales límites cronológicos y que según parece se pueden atribuir a Constante: CTh 11, 36, 4 (29 agosto 339), CTh 12, I, 26 (1 noviembre 338), CTh 12, 1, 27 (8 enero 339), CTh 12, 1, 28 (26 noviembre 339) (BARNES, 1980, 164-166; SEECK, 1919, 184 ss.). En consecuencia, la primera exigencia de J. R. Palanque se cumple.

Con respecto a las otras dos condiciones impuestas sólo hay un método válido que permite su comprobación: el prosopográfico.

En efecto, gracias a la evidencia prosopográfica se aprecia un hecho indiscutible: el que no se conoce ningún magister officiorum, quastor palatii, comes rei privatae ni comes largitionum de ninguno de los tres hermanos durante este trienio, y los que se registran de Constante y Constancio II son únicamente de una fecha posterior al 340 (PLRE I, Fasti 1060-1064).

Por lo tanto, opino que la circunstancia de que no nos haya llegado el nombre de ningún «ministro» de Constante, perteneciente a las fechas 337-340, no puede considerarse como un argumento decisivo en contra de la soberanía de este augusto, y ello por dos razones: la primera, es porque tampoco ha llegado el nombre de ninguno de los ministros de Constantino II y nadie duda de su autoridad; incluso se le llega a adjudicar un poder superior que al resto de los emperadores. La segunda razón es que con Constancio II se repite la misma situación que la de Constante; los funcionarios que se le conocen ejercen el cargo en fechas posteriores al $340 \mathrm{y}$, sin embargo, nadie duda que cuenta con una soberanía efectiva desde el 337.

Seguidamente se ve que el método prosopográfico vuelve a ser determinado en relación a la última condición de $\mathbf{J}$. R. Palanque, la necesidad de que un augusto nombre magistrados para que pueda ser considerado soberano. Pues bien, puedo ciertamente demostrar que tal caso se da, puesto que se registran once nombres de funcionarios nombrados por Constante entre el 337 y el 340: L. Turcio Aproniano (PLRE I, 88 n. 9), Aco Catullino Philomatio (PLRE I, 187 n. 3), Eutherio (PLRE I, 314 n. 1), Munatio Gentiano (PLRE I, 391 n. 2), Maecilio Hilariano (PLRE I, 433 n. 5), Lucio Crepereio Madaliano (PLRE I, 530), Martiniano (PLRE I, 563 n. 3), M. Maecio Memmio Furio Baburio Caeciliano Plácido (PLRE I, 705 n. 2), L. Aradio Valerio Próculo Populonio (PLRE I, 747 n. 11), Fl. Julio Rufino Sarmentio (PLRE I, 804), Anónimo (PLRE I, 1014 n. 51).

En conclusión, creo haber demostrado que se le puede atribuir a Constante una corte y una legislación desde el 337. De ello se deduce, según las condiciones impuestas por J. R. Palanque, que el hijo menor de Constantino obtuvo plenos poderes soberanos desde aquella fecha, al igual que sus hermanos.

Una vez comprobado que Constante no se encontraba bajo la tutela de su hermano mayor Constantino II, debe observarse el conflicto que estalla entre ambos bajo una perspectiva diferente a como se ha venido haciendo hasta ahora. En efecto, el enfrenta- 
miento armado se desencadena en el 340 (14). Se ha interpretado que ello fue debido a una rebelión de Constante, quien quería librarse de la tutela de su hermano (15); sin embargo, el fundamento de esta suposición ha sido rebatido más arriba, por lo cual, ella misma queda desmentida. Lo cierto es que la provocación procedió de Constantino II (BARNES, 1980, 162 n. 9; BARNES, 1981, 263) porque no se conformó con ocupar una posición superior exclusivamente honorífica, sino que deseó hacer efectiva dicha preeminencia; es decir, quiso implantar su autoridad en todo Occidente; por ello, Constantino II invadió Italia, territorio de su hermano, lo cual es otro dato a favor de que la responsabilidad fue de Constantino y no de Constante, pues éste ni siquiera se encontraba allí, sino que estaba en Naiso (16) y la defensa se improvisó con las fuerzas que estaban allí estacionadas. A partir de ese momento el desarrollo de los acontecimientos es bien conocido; el ejército fiel a Constante hace frente al invasor, Constantino II, quien muere en Aquileya; cuando Constante llega a Italia todo había concluido (17).

(14) Son diversas las fuentes que informan sobre este hecho: AUR. VICT. Epit. 41, 21; AUR. VICT. Caes. 41, 22; Chron. Min. 1, 236; EUTR. Brev. 10, 9, 2-4; HIER. Chron. 2356; JUL. Or. 2, 94 B; PHILOST. $H E$ 3, 1 a; SOCR. $H E$ 2, 5; SOZ. 3, 2; ZON. 13, 5, 5-9; ZOS. 2, 41.

(15) Opinión expuesta en ZOS. 2, 41; cfr. PALANQUE, 1944, 58 ss.; MOREAU, 1959, 179; SEECK, 1901, 949; PIGANIOL, $1972^{2}, 84$.

(16) Hay abundantes testimonios de ello: CTh 2, 6, 5; 10, 10, 5; 10, 15, 3; 12, 1, 29; 12, 9, 29; ZON. $13,5,9$.

(17) Véase nota 14; cfr. BARNES, 1982, 86-87. 


\section{FUENTES}

AMMIANUS MARCELLINUS, 1978, ed. W. Seyfarth, Teubner. L'ANNÉ ÉPIGRAPHIQUE.

ANONYMUS VALESIANUS, 1874, ed. V. Gardthausen, Teubner.

ATHANASIO, Patrologia Graeca, vols. 25-26.

AUGUSTIN, contra Cresconium, ed. Petschenig, CSEL, 52.

AURELIO VICTOR, 1961, de Caesaribus, ed. Pichlmayer, Teubner.

AUSONIO, 1968, Works, ed. E. White, Londres.

CHRON. MIN., 1892, Chronica Minora, Monumenta Germanicae Historica, AA 9, ed. Th. Mommsen, Berlín.

CHRONICON PASCHALE, 1832, ed. L. Dindorf, Bonn.

CODEX IUSTINIANUS, 1967 (reimp.), ed. P. Krüger, Berlín.

CODEX THEODOSIANUS, 1971 (reimp.), ed. Th. Mommsen, Berlín.

CORPUS INSCRIPTIONUM LATINARUM, 1864-1943, ed. W. de Gruyter, Berlín.

EUNAPIO, 1894, Vitae Sophistarum, ed. Boissonade.

EUSEBIO, 1975, de vita Constantini, ed. F. Winkelmann, GCS, Berlín. Laudes Constantini, PG, vol. 20.

EUTROPIO, 1965, Breviarium ab urbe condita, ed. B. Calvi, Turín.

FIRMICO MATERNO, 897-1913, ed. W. Kroll - F. Skutsch, Teubner.

FRAGMENTA VATICANA, FIR ${ }^{2}$ II, 461-540.

HIERONIMUS, 1965, Eusebius Werke 7: Die Chronik des Hieronymus, ed. R. Helm, GCS 47, Berlín., 1924, de viris illustribus, ed. Herding.

HILARIO, 1916, ed. Feder, CSEL, t. LXV.

INSCRIPTIONES LATINAE SELECTAE, 1892-1916, ed. H. Dessau, Berlín.

INSCRIPTIONS LATINES DE LA TUNISIE, JUAN CRISOSTOMO, Patrologia Graeca. París.

JULIANO, 1924-1932, Oeuvres Complètes, ed. J. Bidez, París.

LIBANIO, 1903-1922, Orationes, ed. R. Foerster, Teubner.

MALALAS, 1831, Chronographia, ed. Dindorf, Bonn.

MANSI, 1960, Sacrorum Conciliorum nova et amplissima collectio, Graz.

OPTATUS, 1893, ed. K. Ziwsa, CSEL 26, Viena.

P. LOND, 1893-1917, Greek Papyri in the British Museum, ed. F. G. Kenyon. H. I. Bell, Londres.

THE OXYRHYNCHUS PAPYRI, 1898, ed. B. P. Grenfell y otros, Londres.

PALLADIO, 1928, Historia Lausiaca, PG, ed. Norton.

PANEGYRICI LATINI, 1952, ed. Galletier, París.

PHILOSTORGO, 1972, Kirchengeschichte, ed. Bidez (2. ${ }^{a}$ ed. F. Winkelmann) GCS, Berlín.

ROMAN IMPERIAL COINAGE, 1920, Londres.

SOCRATES, 1853, Historia eclesiástica, ed. Hussey, Oxford.

SOZOMENO, Historia Eclesiástica, PG 67, 843-1630.

SYMMACO, 1883, Relationes, MGH, AA VI.

SYNESIO, 1893, Epistulae, ed. Hercher.

THEODORETO, 1911, Historia Ecclesiastica, ed. Parmentier, Berlín.

VITA OLYMPIADIS, 1968 , ed. A. M. Marlingrey, $S C, 13$ bis.

ZONARAS, 1868-1875, Epitome Historiarum, es. L. Dindorf, Teubner.

ZOSIMO, 1971-1979, Histoire Nouvelle, ed. F. Paschoud, París. 
ARNHEIM, M. T. W., 1982. The Senatorial aristocracy of the Later Roman Empire. Oxford.

BARNES, T. D., 1974. «Another forty missing persons (A. D. 260-395)», Phoenix, 28, 224-33.

- 1975. "Constans and Gratian in Rome», HSCPh, 79, 325-333.

- 1976. «The victories of Constantine», $Z P E, 20,149-55$.

- 1978. «Emperors and bishops, A. D. 324-44. Some Problems», $A J A H, 3$, 53-75

- 1980. «Imperial Chronology, A. D. 337-350», Phoenix, 34, 160-66.

- 1981. Constantine and Eusebius, Harvard.

- 1982. The New Empire of Diocletian and Constantine, Harvard.

BRUUN, P., 1966. Roman Imperial Coinage VII, A. D. 313-337. Londres.

BURCKHARDT, J. 1982 (reimpresión). Del paganismo al cristianismo. La época de Constantino el Grande. Madrid.

COHEN, H. 1955. Description historique des monais frapées sous l'Empire romain, Graz.

CHASTAGNOL, A. 1968. "Les préfets du prétoire de Constantin», REA, 70, 321-52.

- 1969. Le Bas-Empire. París.

- 1976. «Les inscriptions constantiniennes du Cirque de Mérida», MEFR 88, 259-76.

- 1982. L'évolution politique, sociale et economique du Monde Romain de Dioclétien à Julien. La mise en Place du Régime du Bas-Empire (284-363). París.

DEMOUGEOT, E. 1979. «Le partage des provinces de l'Illyricum entre la pars occidentis et la pars orientis, de la Tétrarchie au regne de Théodosio». Actes du Colloque de Strasbourg 14-16 juin 1979. La Géographie administrative et politique d'Alexandre à Mahomet, 229-253.

FORTINA, M. 1955. La legislazione dell'imperatore Costante. Novara.

GALLETIER, E. 1952. Panégyriques Latins. París.

GARCIA IGLESIAS, L. 1979. «Paganismo y cristianismo en la España romana». La Religión romana en Hispania (Symposio organizado por el Inst. de Arqueología «Rodrigo Caro» del C. S. I. C. del 17 al 19 de diciembre de 1979). Madrid, 363-79.

GARRIDO GONZALEZ, E. 1983. Los gobernadores provinciales en el siglo IV d. C. Estudio de la estructura sociopolitica de Occidente a través de la administración. Madrid (Tesis de doctorado inédita).

- 1984. «Una aproximación al conocimiento de la administración provincial del s. IV: la diócesis de Thracia». AEArq. (en prensa).

GAUDEMET, J. 1979. «Mutations politiques et géographie administrative: L’Empire romain de Dioclétien (284) à la fin du Ve siècle». Actes du Colloque de Strasbourg 14-16 juin 1979. La Geógraphie administrative et politique d'Alexandre à Mahomet. 255-72.

GIGLI, G. 1959. La dinastia dei Secondi Flavii; Costantino II, Costante, Costanzo II (337-361). Roma. HAUSCHILD, Th. 1966. «Die Grabungen in Centcelles», $A A, 86-92$.

HERZ, P. 1975. Untersuchungen zum Festkalender der römischen Kaiserzeit. Mainz.

JONES, A. H. M. 1973. The Later Roman Empire 284-602. A social economic and administrative survey. Oxford. 2. ${ }^{\text {a }}$ edición.

- 1974 (Reimpresión). «The date and value of the Verona List». The Roman Economy. Studies in Ancient Economic and Administrative History (ed.) P. A. Brunt, Oxford, 263-79.

KRAFT, K. 1958. «Die Taten der Kaiser Constans u. Constantius II», JNG, 9.

LIEBESCHUETZ, J. H. W. G. 1979. Continuity and Change in Roman Religion. Oxford.

MARROU, H. I. 1975. Historia de la educación en la Antigüedad. Buenos Aires. 3. a edición.

MAURICE, J. 1911. «La dynastie solaire des seconds Flaviens». $R A$.

MITFORD, J. 1939. «Milestones in western Cyprus». JRS, 29, 184-198.

MOREAU, J. J. 1959. «Constans, Constantius II, Constantinus II», JbAC 2, 179-84.

OLIVETTI, A. 1915. "Sulle stragi di Constantinopoli succedute alla morte di Costantino il grande» Riv. Fil. 43.

PALANQUE, J. R. 1938. «Chronologie Constantinniene». REA, 40, 241-50.

- 1944. «Collégialité et partages dans l'Empire romain aux IVe et Ve siècles». $R E A, 46,27-64$.

- 1955. «Les préfets du prétoire sous les fils de Constantin». Historia, 4, 257-63.

PETIT, P. 1974. Histoire Générale de l'Empire romain. París.

PIGANIOL, A. 1972. L'Empire Chrétien. París. 2. ${ }^{\text {a }}$ edición.

REMONDON, R. 1973. La crisis del Imperio Romano. De Marco Aurelio a Anastasio. Barcelona.

ROBERTSON, A. S. 1982. Roman Imperial Coins in the Hunter coin cabinet. University of Glasgow. V. Diocletian (Reform) to Zeno. Oxford.

SAYAS, J. J., GARCIA MORENO, L. A. 1981. Romanismo y Germanismo. El despertar de los pueblos hispánicos. Barcelona.

SEECK, O. 1898. «Zu den Festmünzen Constantius und seiner Familie». ZNum., 21, 17-65.

- 1895-1921. Geschichte des Untergangs der Antiken Welt. Stuttgart. 
- 1901. «Constans», $P W 4,948-52$.

- 1919. Regesten der Kaiser and Päpste für die Jahre 311 bis 476 n. Chr., Stuttgart.

STEIN, E. 1959. Histoire du Bas-Empire. París.

THIRION, M. 1965. «Les vota impériaux sur les monnaies entre 337 at $364 » S N R, 44,5-21$.

THOMPSON, E. A. 1956. "Constantine, Constantius II and the lower Danube frontier». Hermes, 84, 372-81.

TOYNBEE, J. M. C. 1944. Roman Medallions. New York.

VOGLER, C. 1979. Constance II. Estrasburgo. 